\title{
SYSTEMATIC OF TauSpinner FOR $\tau$ PAIRS WITH TWO HARD JETS AND ITS RECENT DEVELOPMENT*
}

\author{
M. BAhmani ${ }^{\mathrm{a}}$, J. KALinOWSKi ${ }^{\mathrm{b}}$, W. Kotlarski ${ }^{\mathrm{b}, \mathrm{c}}$ \\ E. RICHTER-WĄ ${ }^{d}$, Z. WĄ $S^{a}$
}

${ }^{a}$ The H. Niewodniczański Institute of Nuclear Physics, Polish Academy of Sciences Radzikowskiego 152, 31-342 Kraków, Poland

${ }^{\mathrm{b}}$ Faculty of Physics, University of Warsaw, Pasteura 5, 02-093 Warszawa, Poland ${ }^{\mathrm{c}}$ Institute for Nuclear and Particle Physics Zellescher Weg 19, 01069 Dresden, Germany

${ }^{\mathrm{d}}$ The M. Smoluchowski Institute of Physics, Jagiellonian University Łojasiewicza 11, 30-348 Kraków, Poland

\section{(Received March 28, 2017)}

In this paper, we review results obtained by using the TauSpinner package. A sample of $p p \rightarrow \tau \tau+j j$ events, where spin effects are not included, is supplemented with weight, then helicities of the $\tau$ leptons are attributed. The systematic uncertainties of TauSpinner due to variation of its input parameters as well as its recent development on implementation of nonStandard Model matrix elements are presented.

DOI:10.5506/APhysPolB.48.903

\section{Introduction}

The $\tau$ lepton offers a powerful probe in many areas, such as studies of hard process characteristics, measurements of properties of Higgs boson or in search for new physics [1,2]. The TauSpinner package [3] is a tool that allows to modify the physics model of Monte Carlo generated samples containing $\tau$ lepton. Information on polarization and spin correlation are reconstructed from the kinematics of $\tau$ leptons $(\tau$ lepton production and decay), and the information on initial state partons is assumed to be unknown. In order to calculate the spin weight, assumed PDF (parton distribution function) configuration and matrix element for the hard process are used. In this paper, in Section 2, we present the new features of TauSpinner package for $2 \rightarrow 4$ parton level process documented in [4], and available

* Presented by M. Bahmani at the Cracow Epiphany Conference "Particle Theory Meets the First Data from LHC Run 2", Kraków, Poland, January 9-12, 2017. 
from http://tauolapp.web.cern.ch/tauolapp/. Then, in Section 3, we discuss systematic uncertainties of TauSpinner due to variation of its input parameters. Section 4 focuses on the interference term between QCD and EW production for ME calculation. Finally, Section 5 introduces recent developments on the non-Standard Model matrix element implementation.

\section{Features of TauSpinner program in $2 \rightarrow 4$ parton level process}

The physics processes of interest for matrix element implementation are Standard Model processes in $p p$ collision with two opposite-sign $\tau$ leptons and 2 jets in the final state. The method is based on tree-level matrix elements with complete helicity information for Standard Model processes. In the new version of TauSpinner, one has the freedom to change the input parameters by using the initialization for QCD factorization and renormalization scale and Electroweak scheme. Anomalous contributions can be provided by the user, as well.

\subsection{Electroweak scheme}

In the process of calculating the spin weight, in the case of $2 \rightarrow 2$ process, the main implemented option is an effective Electroweak scheme, using the lowest order ME for the $q \bar{q} \rightarrow Z / \gamma^{*} \rightarrow \tau \tau$ process, with the effective value for the $\sin \left(\theta_{w}^{\text {eff }}\right)$ and running of $Z$ boson width. In the case of $2 \rightarrow 4$ processes, more options are available [3].

Depending on the initial state, tree-level matrix elements are of the order of $\alpha_{\mathrm{s}} \alpha_{\mathrm{EW}}$ or $\alpha_{\mathrm{EW}}^{2}$, sometimes involving triple $W W Z$ coupling. Therefore, one can either include dominant EW loop corrections simultaneously with QCD corrections or adopt an effective scheme which would allow at tree level to account correctly the $\tau$ lepton polarization at the $Z$ boson peak and physical $W$ boson mass. There are four EW schemes implemented in

TABLE I

EW scheme implemented in TauSpinner ME for processes $(2 \rightarrow 4)$, see [3] for details.

\begin{tabular}{l|l|l|l|l}
\hline \hline \multirow{2}{*}{ Type } & $\begin{array}{l}\text { EWSH }=1 \\
\text { input: } \\
G_{\mathrm{F}}, \alpha_{\mathrm{QED}}, M_{z}\end{array}$ & $\begin{array}{l}\text { EWSH }=2 \\
\text { input: } \\
G_{\mathrm{F}}, \sin ^{2}\left(\theta_{w}\right), M_{z}\end{array}$ & $\begin{array}{l}\text { EWSH }=3 \\
\text { input: } \\
G_{\mathrm{F}}, M_{W}, M_{z}\end{array}$ & $\begin{array}{l}\text { EWSH }=4 \\
\text { input: } \\
G_{\mathrm{F}}, M_{W}, M_{z}, \sin ^{2}\left(\theta_{w}\right)\end{array}$ \\
\hline$M_{z}$ & $91.1882 \mathrm{GeV}$ & $91.1882 \mathrm{GeV}$ & $91.1882 \mathrm{GeV}$ & $91.1882 \mathrm{GeV}$ \\
$M_{W}$ & $80.4190 \mathrm{GeV}$ & $79.9407 \mathrm{GeV}$ & $80.4189 \mathrm{GeV}$ & $80.4189 \mathrm{GeV}$ \\
$\sin ^{2}\left(\theta_{w}\right)$ & 0.222246 & 0.231470 & 0.222246 & 0.231470 \\
$1 / \alpha_{\mathrm{QED}}$ & 132.5070 & 128.7538 & 132.5069 & 127.2272 \\
$G_{\mathrm{F}}$ & $1.6639 \times 10^{-5}$ & $1.6639 \times 10^{-5}$ & $1.6639 \times 10^{-5}$ & $1.6639 \times 10^{-5}$ \\
& $\mathrm{GeV}^{-2}$ & $\mathrm{GeV}^{-2}$ & $\mathrm{GeV}^{-2}$ &
\end{tabular}


TauSpinner $2 \rightarrow 4$, which can be seen in Table I. The first three have 3 input parameters, while the EWSH $=4$ has four input parameter. Since $\tau$ polarization is sensitive to the value of mixing angle and for both Tauola and TauSpinner the $\tau$ physics is an important target, it is recommended to use $\mathrm{EWSH}=4$.

\section{2. $Q C D$ factorization and re-normalization scales and strong coupling constant}

The default implementation imposes QCD scales, factorization and renormalization to be equal $\mu_{\mathrm{F}}=\mu_{\mathrm{R}}=Q^{2}$. For the $2 \rightarrow 4$ process, several choices are possible as specified below:

$$
\begin{array}{ll}
\text { scalePDFOpt }=0 & Q=200 \mathrm{GeV}, \\
\text { scalePDFOpt }=1 & Q=\sqrt{\widehat{s}}, \\
\text { scalePDFOpt }=2 & Q=\sum m_{\mathrm{T}}, m_{\mathrm{T}}^{2}=m^{2}+p_{\perp}^{2}, \\
\text { scalePDFOpt }=3 & Q=\sum E_{\perp}, E_{\perp}=E_{P_{\perp}} /|\vec{p}|
\end{array}
$$

As a default, the scalePDFOpt=1 option is considered. For the $\alpha_{\mathrm{s}}\left(Q^{2}\right)$, a simple choice of the $Q^{2}$ dependence is provided by the following leading logarithmic formula:

$$
\alpha_{\mathrm{s}}\left(Q^{2}\right)=\frac{\alpha_{\mathrm{s}}\left(M_{Z}^{2}\right)}{1+4 \pi \alpha_{\mathrm{S}}\left(M_{Z}^{2}\right)\left(11-2 N_{\mathrm{f}} / 3\right) \ln \frac{Q^{2}}{M_{Z}^{2}}}
$$

with the starting point $\alpha_{\mathrm{s}}\left(M_{Z}^{2}\right)=0.118$. The same value of $\alpha_{\mathrm{s}}$ is used for the case of the fixed coupling constant, that is for scalPDFOpt $=0$.

In the LHAPDF package which is used for providing parameterization of the structure functions, the calculation of $\alpha_{\mathrm{S}}$ is implemented for each set of parameterization. It is, therefore, possible to use the definition of the strong coupling consistent with the choice of the structure functions parameterization.

\subsection{Parton density function}

For calculating spin weight, TauSpinner is using the average of the production cross section for different flavors of incoming partons, weighted according to the assumed PDFs. The code of TauSpinner is interfaced to LHAPDF library, therefore, any other parameterization of parton density function is easy to install. 


\section{Systematic uncertainties}

In this section, we discuss systematic uncertainties on the average $\tau$ lepton polarization and other sensitive observables such as visible energy fraction in the $\tau \rightarrow \pi \nu$ decays. For the numerical results discussed here, we use long series (about $10^{6}$ ) of Drell-Yan events of $p p \rightarrow \tau \tau+j j$ process generated with the MadGraph package [6]. Table II gives the average $\tau$-lepton polarization with different EW schemes used for spin weight calculations for $2 \rightarrow 2$ and $2 \rightarrow 4$ matrix elements. Figure 1 shows the distribution of the visible energy fraction in the $\tau \rightarrow \pi \nu$ decays, which is sensitive to polarization. One can clearly notice the impact on the average polarization and sensitive observables due to the different EW schemes.

TABLE II

$\tau$-lepton polarization in the window $88<M_{\tau \tau}<92 \mathrm{GeV}$ for different EWSH.

\begin{tabular}{l|c|c|c|c}
\hline \hline & EWSH $=1$ & EWSH $=2$ & EWSH $=3$ & EWSH $=4$ \\
\hline $2 \rightarrow 4$ & $-0.2048 \pm 0.0015$ & $-0.1385 \pm 0.0015$ & $-0.2014 \pm 0.0015$ & $-0.1331 \pm 0.0015$ \\
$2 \rightarrow 2$ & $-0.2063 \pm 0.0015$ & $-0.1407 \pm 0.0015$ & $-0.2055 \pm 0.0015$ & $-0.1412 \pm 0.0015$
\end{tabular}
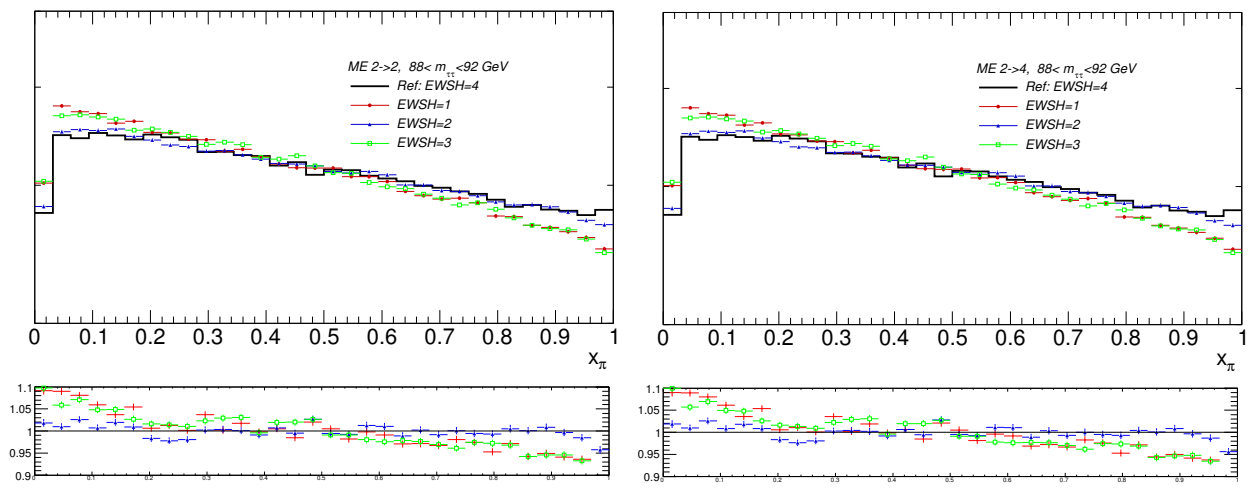

Fig. 1. Visible energy fraction in the $\tau \rightarrow \pi \nu$ decays for different EW schemes for $2 \rightarrow 2$ and $2 \rightarrow 4$ matrix elements.

In Tables III and IV, the average $\tau$-lepton polarization with different QCD scale option and different PDF sets which is used for spin weight calculations for $2 \rightarrow 2$ and $2 \rightarrow 4$ matrix elements are shown, respectively. In both cases, one can see that there is no significant difference between the result calculated from $2 \rightarrow 2$ and $2 \rightarrow 4 \mathrm{ME}$. The variation of TauSpinner results with respect to different QCD scale option is 3.6 percent and 1.7 percent with respect to different PDF sets. 
$\tau$-lepton polarization in window $88<M_{\tau \tau}<92 \mathrm{GeV}$ for different QCD scales.

\begin{tabular}{l|l|l|l|l}
\hline \hline & scalePDFOpt $=0$ & scalePDFOpt $=1$ & scalePDFOpt = 2 & scalePDFOpt = 3 \\
\hline $2 \rightarrow 4$ & $-0.1362 \pm 0.0015$ & $-0.1331 \pm 0.0015$ & $-0.1380 \pm 0.0015$ & $-0.1363 \pm 0.0015$ \\
$2 \rightarrow 2$ & $-0.1368 \pm 0.0015$ & $-0.1412 \pm 0.0015$ & $-0.1377 \pm 0.0015$ & $-0.1378 \pm 0.0015$
\end{tabular}

TABLE IV

$\tau$-lepton polarization in the window $88<M_{\tau \tau}<92 \mathrm{GeV}$ for different PDF sets.

\begin{tabular}{l|l|l}
\hline \hline \multicolumn{1}{c|}{ PDF sets } & TauSpinner $2 \rightarrow 4$ & TauSpinner $2 \rightarrow 2$ \\
\hline cteq611.LHpdf & $-0.1331 \pm 0.0015$ & $-0.1412 \pm 0.0015$ \\
MSTW2008nnlo90cl.LHgrid & $-0.1354 \pm 0.0015$ & $-0.1379 \pm 0.0015$ \\
CT10.LHgrid & $-0.1338 \pm 0.0016$ & $-0.1368 \pm 0.0016$
\end{tabular}

\section{Interference between QCD and electroweak sub-processes}

In TauSpinner, depending on initial state, the matrix elements are of the order of $\alpha_{\mathrm{s}} \alpha_{\mathrm{EW}}$ or $\alpha_{\mathrm{EW}}^{2}$. In MadGraph for generating ME, parameter QED is set to 4 which enforces diagrams up to $4^{\text {th }}$ order in the electroweak couplings. In the case of other setting, one can check results also for the second order of electroweak couplings. In such a case, some of the diagrams which include QCD couplings will be eliminated, however, one should check if there is any significant interference between those diagrams and EW diagrams. In the case of Standard Model, the square of matrix element is calculated from formula (2), without QCD contribution, the first and last terms in this formula will vanish. However, if $\alpha_{\mathrm{S}}$ is set to be large, one must check if the last terms has any effect in the ME calculation. The matrix element squared is calculated by the following formula:

$$
\begin{aligned}
|\mathrm{ME}|^{2} & =\left|\alpha_{\mathrm{s}} \alpha_{\mathrm{EW}} M_{1}+\alpha_{\mathrm{EW}}^{2} M_{2}\right|^{2} \\
& =\left|\alpha_{\mathrm{s}} \alpha_{\mathrm{EW}} M_{1}\right|^{2}+\left|\alpha_{\mathrm{EW}}^{2} M_{2}\right|^{2}+\alpha_{\mathrm{EW}}^{3} \alpha_{\mathrm{S}}\left(M_{1} M_{2}^{*}+M_{1}^{*} M_{2}\right) .
\end{aligned}
$$

The aim of this check is to quantify the interference term (third term in Eq. (2)) between QCD and EW production for ME calculation. TauSpinner allows to calculate weight which is a ratio of non-SM/SM cross section. For non-SM, we set $\alpha_{\mathrm{QCD}}$ to be $0, \alpha_{\mathrm{S}}$ and $2 \alpha_{\mathrm{S}}$ for different energy scale in non$\mathrm{SM}$ calculation. For the SM, the nominal value is used. In the case of $\alpha_{\mathrm{QCD}}=0$, only EW diagrams contribute with $Z \rightarrow \tau \tau$ and $W \rightarrow q \bar{q}$ leading to $\tau \tau+j j$ in the final state. So, we will get the cross section of the EW diagrams only in the ME calculation. We cannot set $\alpha_{\mathrm{QED}}=0$, but as the 
$\alpha_{\mathrm{S}}$ is much larger than $\alpha_{\mathrm{QED}}$, setting for non-SM the option of $\alpha_{\mathrm{QCD}}=2 \alpha_{\mathrm{S}}$ in calculation of ME strongly enhances the contribution of QCD diagrams with respect to EW ones. In the presented test, two MadGraph samples were used, the first one had cut on invariant mass of leptons at the generation level $\left(30 \mathrm{GeV}<M_{l l}<160 \mathrm{GeV}\right)$. The second one had kinematical cuts both at the generation and analysis level, which are shown in Table V.

TABLE V

Kinematical selection for $Z \rightarrow \tau \tau+j j$ samples at generation and analysis level.

\begin{tabular}{l}
\hline Selection at analysis level \\
\hline$P_{\mathrm{t}}$ of jets $>50 \mathrm{GeV}$ \\
$P_{\mathrm{t}}$ of jet1 $>30 \mathrm{GeV}$ or $P_{\mathrm{t}}$ of jet2 $>30 \mathrm{GeV}$ \\
$\eta_{j 1}-\eta_{j 2}>3.0$ \\
$P_{\mathrm{t}}$ of $\tau 1>25 \mathrm{GeV}$ or $P_{\mathrm{t}}$ of $\tau 2>25 \mathrm{GeV}$ \\
$P_{\mathrm{t}}$ of $\tau \mathrm{s}>35 \mathrm{GeV}$ \\
$\left|\eta_{\tau 1}\right|<2.5$ or $\left|\eta_{\tau 2}\right|<2.5$ \\
\multicolumn{1}{c}{ Selection at generation level } \\
\hline$P_{\mathrm{t}}$ of jets $>50 \mathrm{GeV}$ \\
$P_{\mathrm{t}}$ of leptons $>30 \mathrm{Gev}$ \\
Invariant mass of jets $>120 \mathrm{GeV}$ \\
Invariant mass of leptons $>120 \mathrm{GeV}$
\end{tabular}

For quantifying the interference term with simple math, one can see that for

$$
\begin{aligned}
\alpha_{\mathrm{QCD}} & =\alpha_{\mathrm{s}}: \\
\sigma^{\mathrm{tot}} & =\sigma^{\mathrm{QCD}}+\sigma^{\mathrm{EW}}+\sigma^{\mathrm{I}}=|\mathrm{ME}|^{2}=\left|\alpha_{\mathrm{EW}}\left(\alpha_{\mathrm{s}} M_{1}+\alpha_{\mathrm{EW}} M_{2}\right)\right|^{2} \\
& =\left.\alpha_{\mathrm{s}} \alpha_{\mathrm{EW}} M_{1}\right|^{2}+\left|\alpha_{\mathrm{EW}}^{2} M_{2}\right|^{2}+\alpha_{\mathrm{EW}}^{3} \alpha_{\mathrm{s}}\left(M_{1} M_{2}^{*}+M_{1}^{*} M_{2}\right), \\
\alpha_{\mathrm{QCD}} & =2 \alpha_{\mathrm{s}}:|\mathrm{ME}|^{2}=4 \sigma^{\mathrm{QCD}}+\sigma^{\mathrm{EW}}+2 \sigma^{I}, \\
\alpha_{\mathrm{QCD}} & =0:|\mathrm{ME}|^{2}=\left|\alpha_{\mathrm{EW}}^{2} M_{2}\right|^{2}=\sigma^{\mathrm{EW}} .
\end{aligned}
$$

The interference term $\sigma^{\mathrm{I}}$ can be expressed as the function of cross section, and its ratio to nominal cross section is given by

$$
\frac{\sigma^{\mathrm{I}}}{\sigma^{\text {tot }}}=\frac{1}{2}\left[4-3 \frac{\sigma_{\alpha=0}^{\mathrm{QCD}}}{\sigma^{\mathrm{tot}}}-\frac{\sigma_{2 \alpha_{\mathrm{s}}}^{\mathrm{QCD}}}{\sigma_{\text {tot }}}\right] .
$$

According to the results shown in Table VI, the interference term for the first sample without cut is one percent and for the case of second sample with kinematical cuts, it is around two percent, which can be neglected. 
The cross section for different $\alpha_{\mathrm{QCD}}$ used for ME calculation, and fraction of the interference term for two different samples.

\begin{tabular}{l|c|c}
\hline \hline Sample without cut & $\sigma(\mathrm{pb})$ & $\left(\sigma^{\mathrm{I}} / \sigma^{\text {tot }}\right)$ \\
\hline$\alpha^{\mathrm{QCD}}=\alpha_{\mathrm{s}}$ & $474581 \pm 688.8$ & 0.0101 \\
$\alpha^{\mathrm{QCD}}=2 \alpha_{\mathrm{s}}$ & $1897110 \pm 2753.9$ & 0.0202 \\
$\alpha^{\mathrm{QCD}}=0.0$ & $416.694 \pm 7.693$ & 0.0 \\
\hline Sample with cuts & $\sigma(\mathrm{pb})$ & $\left(\sigma^{\mathrm{I}} / \sigma^{\text {tot }}\right)$ \\
\hline$\alpha^{\mathrm{QCD}}=\alpha_{\mathrm{s}}$ & $120602 \pm 347.27$ & -0.0185 \\
$\alpha^{\mathrm{QCD}}=2 \alpha_{\mathrm{s}}$ & $437487 \pm 1272.2$ & -0.037 \\
$\alpha^{\mathrm{QCD}}=0.0$ & $16461.9 \pm 76.69$ & 0.0
\end{tabular}

\section{New development of TauSpinner}

The evidence of a new particle with mass $125 \mathrm{GeV}$ decaying into a pair of $\tau$ leptons at the LHC generates the interest in ascertaining its spin properties. Introducing non-standard states and couplings and studying their effects in the vector-boson-fusion processes and exploiting the spin correlations of $\tau$-lepton pair decay products can be useful. This is why in the new version of TauSpinner, we introduce a possibility to implement external matrix element for non-Standard Model theories. For this purpose, an example directory is created under TauSpinner which user can implement and modify the ME through the example code.

\subsection{Spin two particle matrix element implementation}

We start by extending the Lagrangian of Ref. [4] by a set of dimension 5 operators, coupling the spin two field $X$ to gauge boson field strength tensors $B, W$ and $G$ as

$$
\mathcal{L} \ni \frac{1}{F} X_{\mu \nu}\left(g_{X B B} B^{\mu \rho} B_{\rho}^{\nu}+g_{X W W} W_{i}^{\mu \rho} W_{\rho}^{\nu}+g_{X g g} G^{\mu \rho} G_{\rho}^{\nu}\right) .
$$

The scale $F=1 \mathrm{TeV}$. This extension of the SM by a spin two field, including its coupling to quarks and tau leptons, is encoded into FeynRules [5] model. This is done with the following commands in MadGraph:

(a) import model spin2_w_CKM_UFO

(b) with default definition of multiparticles

$$
\begin{aligned}
& p=g u c d s u^{\sim} c^{\sim} d^{\sim} s^{\sim} \\
& j=g u c d s u \sim c^{\sim} d^{\sim} s^{\sim}
\end{aligned}
$$


(c) for the spin two signal processes

generate $\mathrm{p} \mathrm{p}>$ j j $\mathrm{x}$ QED $<=99$ QCD $<=99 \mathrm{NPgg}<=99 \mathrm{NPqq}<=99$ NPVV $<=99, x>$ ta + ta-

(d) and print the output using output standalone "directory name",

where NPgg controls the maximum number of $g_{X g g}$ couplings, NPqq the maximum number of $g_{X q \bar{q}}$ couplings and NPVV the total maximum number of $g_{X W W}, g_{X B B}$ couplings. Setting them to 99, effectively means that their number is not restricted. The model includes the CKM matrix in Wolfenstein parameterization. The generated codes for the individual sub-processes are grouped into subroutines. Before integrating these subroutines into the TauSpinner program, a number of modifications have been done similarly to what is explained in [3] for $2 \rightarrow 4$ process. For the purpose of testing the consistency of the implemented codes generated with MadGraph5 and modified as explained above, we have chosen a fixed kinematic configuration at the parton level. For such kinematics, we have calculated the matrix element squared for all possible helicity configurations of all sub-processes using the codes implemented in TauSpinner and checked against the numerical values obtained directly from MadGraph5. The agreement of at least six significant digits has been confirmed.

\subsection{Tests of matrix elements using series of generated events}

As a further test of the internal consistency of the matrix element implementation in TauSpinner, we have used the reweighting procedure by comparing a number of kinematical distributions obtained directly from a series of 19 million events generated by MadGraph for spin two Higgs-like particle and Higgs with the width of $4 \mathrm{MeV}$. In Fig. 2, the $\Delta R_{j j}$ distribution (angu-
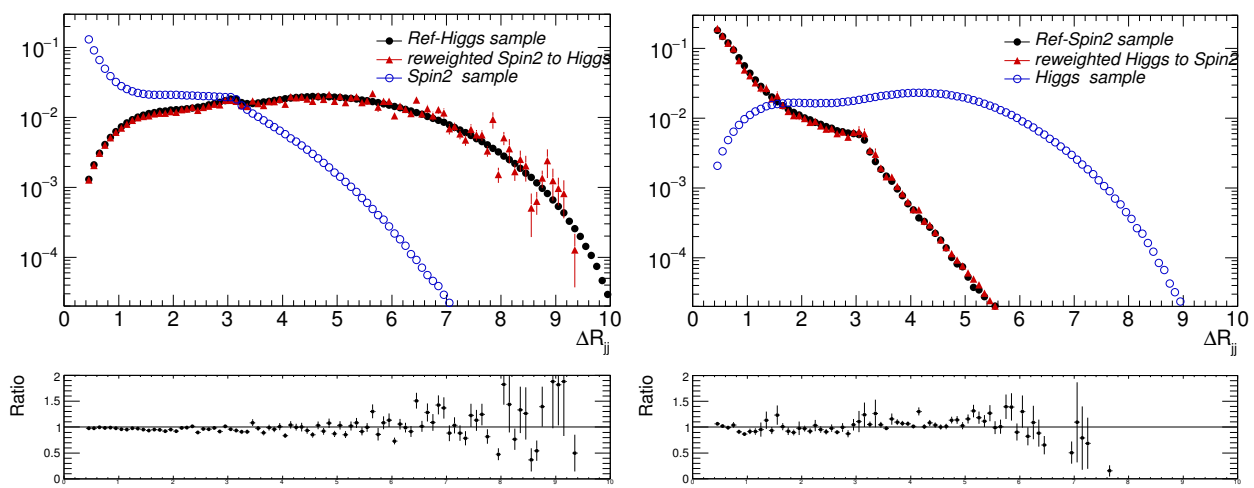

Fig. 2. Distributions of the $\Delta R_{j j}$ for spin two sample reweighted to Higgs and vice versa. 
lar distance between jets) is shown, with cuts on invariant mass of outgoing particles $<1500 \mathrm{GeV}$, invariant mass of jets $<800 \mathrm{GeV}$ and $P_{\mathrm{t}}^{\tau \tau}<600 \mathrm{GeV}$ applied in the case of Higgs sample reweighted to spin two sample.

\section{Summary}

The paper presents the comparison for several options of TauSpinner initialization. The obtained differences can be used to infer systematic errors. Moreover, the results of calculation of interference between QCD and electroweak sub-processes indicate that this term can be neglected. In the last section, we present the recent development of TauSpinner program by introducing non-SM ME. Tests of ME implementation have also been done successfully.

The authors would like to thank for the support from funding agencies. M. Bahmani and Z. Was were supported by European Union under the Grant Agreement PIT- NGA2012316704 (HiggsTools). W. Kotlarski, Z. Wąs and E. Richter-Was were supported by the Polish National Science Centre (NCN) under decisions UMO-2014/15/ B/ST2/00049. Majority of the simulations were performed at PLGrid Infrastructure of the Academic Computer Center CYFRONET AGH in Kraków, Poland.

\section{REFERENCES}

[1] G. Aad et al. [ATLAS Collaboration], J. High Energy Phys. 1504, 117 (2015).

[2] S. Chatrchyan et al. [CMS Collaboration], J. High Energy Phys. 1405, 104 (2014).

[3] J. Kalinowski, W. Kotlarski, E. Richter-Wąs, Z. Wąs, Eur. Phys. J. C 76 , 540 (2016) [arXiv: 1604.00964 [hep-ph]].

[4] S. Banerjee et al., Eur. Phys. C 73, 2313 (2013)

[arXiv:1212.2873 [hep-ph]].

[5] A. Alloul et al., Comput. Phys. Commun. 185, 2250 (2014)

[arXiv:1310.1921 [hep-ph]].

[6] J. Alwall et al., J. High Energy Phys. 1407, 079 (2014)

[arXiv:1405.0301 [hep-ph]]. 


\section{ERRATUM}

(Received August 19, 2017)

\section{Bahmani, J. Kalinowski, W. Kotlarski}

E. Richter-Wąs, Z. Wąs

Systematic of TauSpinner for $\tau$ Pairs With Two Hard Jets and Its Recent Development

Acta Phys. Pol. B 48, 903 (2017)

The following acknowledgment should be added on page 911 :

Paper partially supported by the Polish National Science Center (NCN) under HARMONIA project UMO-2015/18/M/ST2/00518 (2016-2019). 